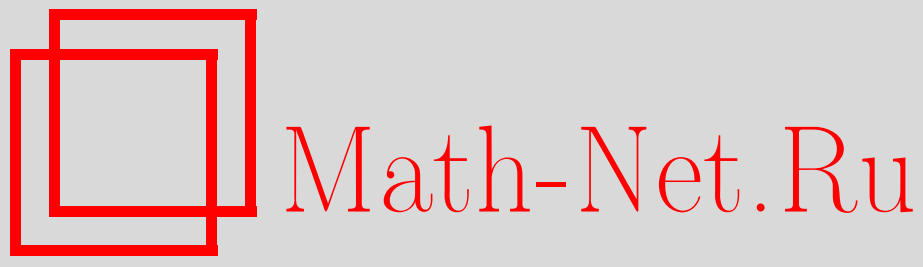

И. Ц. Гохберг, М. А. Каасхук, Л. Е. Лерер, Обратная задача для ортогональных матричных функций Крейна, Функи. анализ и его прил., 2007, том 41, выпуск 2, 44-57

DOI: https://doi.org/10.4213/faa2860

Использование Общероссийского математического портала MathNet.Ru подразумевает, что вы прочитали и согласны с пользовательским соглашением

http://www . mathnet.ru/rus/agreement

Параметры загрузки:

IP : 3.89 .197 .203

26 апреля 2023 г., $17: 36: 19$

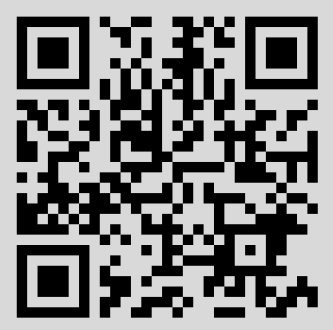




\title{
Обратная задача для ортогональных матричных функций Крейна
}

\author{
(c) 2007. И. ГохБерг, М. А. КАасХУК, Л. ЛЕРеР
}

Памяти М. Г. Крейна - великого математика и превосходного учителя

\section{§1. Введение и основные теоремы}

В цикле работ, начатом в середине пятидесятых годов, М. Г. Крейн ввел и исследовал класс (скалярных) целых функций вида

$$
G_{\omega}(\lambda)=e^{-i \omega \lambda}\left(1+\int_{0}^{\omega} e^{i \lambda s} g(s) d s\right) .
$$

Здесь $\omega-$ положительное число, а $g$ - решение уравнения

$$
g(t)-\int_{0}^{\omega} k(t-s) g(s) d s=k(t), \quad 0 \leqslant t \leqslant \omega,
$$

в пространстве $L_{1}[0, \omega]$, где предполагается, что функция $k$ интегрируема в смысле Лебега на отрезке $[-\omega, \omega]$, а $k(-t)=\overline{k(t)}$. Крейн рассматривал функции $G_{\omega}(\omega>0)$ в качестве континуальных аналогов классических ортогональных многочленов Сегё.

Чтобы увидеть связь с ортогональными многочленами, предположим, что $h$ - положительная функция на единичной окружности $\mathbb{T}$, имеющая абсолютно сходящийся ряд Фурье $\sum_{j=-\infty}^{\infty} h_{j} z^{j}$. При $n=0,1,2, \ldots \quad n$-й классический многочлен Сегё $p_{n}$, определенный функцией $h$, может быть записан в виде

$$
p_{n}(z)=\sum_{\nu=0}^{n} z^{n-\nu} b_{\nu}
$$

где коэффициенты $b_{0}, b_{1}, \ldots, b_{n}$ определяются из уравнения

$$
\left[\begin{array}{cccc}
h_{0} & h_{-1} & \cdots & h_{-n} \\
h_{1} & h_{0} & \cdots & h_{-n+1} \\
\vdots & \vdots & \ddots & \vdots \\
h_{n} & h_{n-1} & \cdots & h_{0}
\end{array}\right]\left[\begin{array}{c}
b_{0} \\
b_{1} \\
\vdots \\
b_{n}
\end{array}\right]=\left[\begin{array}{c}
1 \\
0 \\
\vdots \\
0
\end{array}\right] .
$$

Многочлены $p_{n}(n=0,1,2, \ldots)$ ортогональны относительно скалярного произведения в пространстве $L_{2}(\mathbb{T})$, определяемого с помощью положительной функции $h$ по формуле

$$
\langle f, g\rangle=\frac{1}{2 \pi} \int_{0}^{2 \pi} \overline{g\left(e^{i \theta}\right)} h\left(e^{i \theta}\right) f\left(e^{i \theta}\right) d \theta .
$$


Поскольку предполагается, что функция $h$ положительна на окружности $\mathbb{T}$, то тёплицева матрица $H$ в левой части уравнения (1.3) положительно определена. В непрерывном случае роль матрицы $H$ играет оператор $I-K$, где $K$ - компактный интегральный оператор в $L_{1}[0, \omega]$, входящий в формулу (1.2). Иными словами, формальным аналогом уравнения (1.3) является уравнение

$$
f(t)-\int_{0}^{\omega} k(t-s) f(s) d s=\delta(t), \quad 0 \leqslant t \leqslant \omega .
$$

Чтобы избежать использования $\delta$-функции, сделаем подстановку $g(t)=f(t)-$ $\delta(t)$, после чего уравнение (1.4) перепишется в виде (1.2).

Чтобы получить аналог классических многочленов Сегё $p_{n}(n=0,1,2, \ldots)$ в непрерывном случае, заменим целое число $n$ положительным вещественным числом $\omega$, а многочлен $p_{n}-$ функцией $G_{\omega}$. Далее, положим $z=e^{-i \lambda}$, заменим $z^{(n-\nu)}(0 \leqslant \nu \leqslant n)$ на $e^{-i \lambda(\omega-t)}(0 \leqslant t \leqslant \omega)$ и проинтегрируем от 0 до $\omega$ вместо того, чтобы суммировать от 0 до $n$. Таким образом, аналог многочленов $p_{n}$ в непрерывном случае задается формулой

$$
G_{\omega}(\lambda)=\int_{0}^{\omega} e^{-i \lambda(\omega-t)} f(t) d t=e^{-i \lambda \omega} \int_{0}^{\omega} e^{i \lambda t} f(t) d t .
$$

Как и ранее, делая замену $g(t)=f(t)-\delta(t)$, убеждаемся, что функция $G_{\omega}(\lambda)$ задается формулой (1.1).

В предположении, что оператор $I-K$ положительно определен (что в дискретном случае отвечает положительности функции $h$ ), Крейн [18] показал, что функция $G_{\omega}$ не имеет нулей в замкнутой верхней полуплоскости. Если предположить лишь, что оператор $I-K$ обратим, то у $G_{\omega}$ не будет нулей на вещественной оси и пар сопряженных нулей (см. работы Крейна и Лангера [21], [22]). В этих работах решена также соответствующая обратная задача. А именно, в них показано, что если функция (1.1) не имеет нулей на вещественной оси и пар сопряженных нулей, то существует функция $k \in L_{1}[-\omega, \omega]$, удовлетворяющая условию $k(-t)=\overline{k(t)}$ и такая, что входящая в формулу $(1.1)$ функция $g$ является решением интегрального уравнения (1.2). Дискретный вариант этих результатов, т. е. случай, когда многочлены $p_{n}(z)$ находятся из уравнения $(1.3)$ с вещественной (не обязательно положительной) функцией $h(z)$, был исследован Крейном в работе [20] (см. также [7]).

Заметим, что множитель $e^{-i \omega \lambda}$ в правой части формулы $(1.1)$ не играет роли в упомянутых в предыдущем абзаце результатах. Конкретное значение параметра $\omega$ также несущественно. Поэтому мы опустим множитель $e^{-i \omega \lambda}$ и индекс $\omega$ у $G_{\omega}(\lambda)$ и вместо $G_{\omega}$ будем писать просто $\Phi$.

Новые результаты. Цель данной работы состоит в том, чтобы получить матричные аналоги упомянутых выше результатов Крейна и Лангера. В случае матричных функций наряду с нулями следует учитывать жордановы цепочки и корневые функции. Прежде всего кратко напомним эти понятия.

Пусть $H(\lambda)$ есть $n \times n$-матричная функция, аналитическая в точке $\lambda_{0} \in \mathbb{C}$. Функция $\varphi$ со значениями в $\mathbb{C}^{n}$ называется корневой функиией порядка не ниже $k$ для $H(\lambda)$ в точке $\lambda_{0}$, где $k$ - натуральное число, если (см. [13, разд. А.1]) она аналитична в точке $\lambda_{0}$, вектор $\varphi\left(\lambda_{0}\right)$ отличен от нуля, а функция $H(\lambda) \varphi(\lambda)$ имеет в точке $\lambda_{0}$ нуль порядка не ниже $k$ (т. е. первые $k$ коэффициентов разложения Тейлора функции $H(\lambda) \varphi(\lambda)$ в точке $\lambda_{0}$ нулевые). Отметим, что для 
существования такой корневой функции необходимо и достаточно, чтобы определитель $\operatorname{det} H(\lambda)$ обращался в нуль в точке $\lambda_{0}$. Используя разложения Тейлора

$$
H(\lambda)=\sum_{\nu=0}^{\infty}\left(\lambda-\lambda_{0}\right)^{\nu} H_{\nu}, \quad \varphi(\lambda)=\sum_{\nu=0}^{\infty}\left(\lambda-\lambda_{0}\right)^{\nu} \varphi_{\nu}
$$

функций $H(\lambda)$ и $\varphi(\lambda)$ в точке $\lambda_{0}$, видим, что $\varphi$ является корневой функцией порядка не ниже $k$ для $H(\lambda)$ в точке $\lambda_{0}$ тогда и только тогда, когда

$$
\varphi_{0} \neq 0, \quad \sum_{j=0}^{r} H_{j} \varphi_{r-j}=0, \quad r=0, \ldots, k-1 .
$$

В этом случае будем также говорить, что векторы $\varphi_{0}, \varphi_{1}, \ldots, \varphi_{k-1}$ образуют жорданову цепочку длины $k$ для $H(\lambda)$ в точке $\lambda_{0}$. За дальнейшими подробностями мы отсылаем читателя к [9, разд. 2.1] и [13, разд. А.1].

Зафиксируем теперь обозначения и терминологию. Всюду далее считается, что $\varphi \in L_{1}^{n \times n}[0, \omega]$, и через $\Phi$ обозначается матричная целая функция, задаваемая формулой

$$
\Phi(\lambda)=I+\int_{0}^{\omega} e^{i \lambda t} \varphi(t) d t, \quad \lambda \in \mathbb{C} .
$$

Будем называть ее ортогональной матричной (иелой) функиией Крейна (на отрезке $[0, \omega])$, если в $L_{1}^{n \times n}[-\omega, \omega]$ существует такая эрмитова $n \times n$-матричная функция $k$, что

$$
\varphi(t)-\int_{0}^{\omega} k(t-s) \varphi(s) d s=k(t), \quad 0 \leqslant t \leqslant \omega .
$$

При этом будем также говорить, что функция $\Phi$ порождается функцией $k$. Напомним, что $n \times n$-матричная функция $k$ на отрезке $[-\omega, \omega]$ называется эpмитовой, если $k(t)=k(-t)^{*}$ для всех $t \in[-\omega, \omega]$. Заметим, что в силу результатов из [10] (которые можно также найти в [6, гл. 7] или [14]) из разрешимости уравнения (1.6) вытекает, что оператор $I-K$, определяемый левой частью этого уравнения, обратим в $L_{1}^{n}[0, \omega]$. Таким образом, определение ортогональной функции Крейна неявно содержит требование обратимости оператора $I-K$.

Наш первый основной результат сформулирован в следующей теореме.

Теорема 1.1. Пусть $\varphi \in L_{1}^{n \times n}[0, \omega]$. Определенная формулой (1.5) функиия $\Phi$ есть ортогональная матричная функиия Крейна, порожденная некоторой эрмитовой матричной функичей $k$ из пространства $L_{1}^{n \times n}[-\omega, \omega]$, тогда и только тогда, когда определитель $\operatorname{det} \Phi(\lambda)$ не имеет вещественных нулей, а для любой симметричной пары $\lambda_{0}, \bar{\lambda}_{0}$ нулей этого определителя выполнены соотношения

$$
\sum_{j=0}^{k}\left\langle\varphi_{k-j}, \psi_{j}\right\rangle_{\mathbb{C}^{n}}=0, \quad k=0,1, \ldots, \min \{p, q\}-1,
$$

где $\varphi_{0}, \varphi_{1}, \ldots, \varphi_{p-1}$ и $\psi_{0}, \psi_{1}, \ldots, \psi_{q-1}-$ произвольные жсордановы цепочки для $\Phi$ в точках $\lambda_{0}$ и $\bar{\lambda}_{0}$ соответственно.

В скалярном случае (при $n=1)$ из условия (1.7) при $k=0$ вытекает, что либо $\varphi_{0}=0$, либо $\psi_{0}=0$, либо оба вектора нулевые. Но в жордановой цепочке первый элемент отличен от нуля по определению. Таким образом, в скалярном 
случае соотношения (1.7) эквивалентны условию, что функция $\Phi$ не имеет симметричных пар нулей $\lambda_{0}, \bar{\lambda}_{0}$. Поэтому теорема 1.1 содержит соответствующий результат Крейна и Лангера для скалярных функций.

Необходимое и достаточное условие из теоремы 1.1 может быть сформулировано также в терминах корневых функций следующим образом:

бункиия $\operatorname{det} \Phi(\lambda)$ не имеет вещественных нулей, а для любой симметричной пары $\lambda_{0}, \bar{\lambda}_{0}$ ее нулей выполнено следующее условие: если $\varphi$ - корневая функиия порядка не ниюе р для $\Phi(\lambda)$ в точке $\lambda_{0}, a \psi-$ корневая функиия порядка не ниже q для $\Phi(\lambda)$ в точке $\bar{\lambda}_{0}$, то функиия $\psi(\bar{\lambda})^{*} \varphi(\lambda)$ имеет в точке $\lambda_{0}$ нуль порядка не ниже $\min \{p, q\}$.

Эта альтернативная формулировка будет использована в 3.

Для доказательства теоремы 1.1 свяжем с $\Phi$ матричное функциональное уравнение

$$
U(\lambda) \Phi(\lambda)+\Phi(\bar{\lambda})^{*} V(\lambda)=I_{n}, \quad \lambda \in \mathbb{C} .
$$

Здесь требуется, чтобы неизвестные $U$ и $V$ были $n \times n$-матричными целыми функциями специального вида, а именно,

$$
U(\lambda)=I_{n}+\int_{0}^{\omega} e^{i \lambda t} u(t) d t, \quad V(\lambda)=\int_{\omega}^{2 \omega} e^{i \lambda t} v(t) d t
$$

где $u \in L_{1}^{n \times n}[0, \omega]$ и $v \in L_{1}^{n \times n}[\omega, 2 \omega]$. Будем говорить, что функции $U$ и $V$ образуют регулярную пару решений уравнения (1.8), если они имеют вид (1.9) и удовлетворяют этому уравнению. В следующей теореме сформулирован наш второй основной результат.

Теорема 1.2. Пусть $\varphi \in L_{1}^{n \times n}[0, \omega]$. Функиия Ф вида (1.5) является ортогональной матричной функиией Крейна, порожденной некоторой эрмитовой матричной функиией $k$ из $L_{1}^{n \times n}[-\omega, \omega]$, тогда и только тогда, когда у уравнения (1.8) есть регулярная пара решений $U, V$. При этом для любой регулярной пары решений $U, V$ уравнения (1.8) такую эрмитову матричную функиию $k \in L_{1}^{n \times n}[-\omega, \omega]$ можно получить, сужая на отрезок $[-\omega, \omega]$ обратное преобразование Фуръе матричной функции

$$
\left(U(\bar{\lambda})^{*}-V(\lambda)\right) \Phi(\lambda)^{-1}, \quad \lambda \in \mathbb{R} .
$$

Иными словами, $k=\left.\ell\right|_{[-\omega, \omega]}$, где

$$
\int_{-\infty}^{\infty} e^{i \lambda s} \ell(s) d s=\left(U(\bar{\lambda})^{*}-V(\lambda)\right) \Phi(\lambda)^{-1}, \quad \lambda \in \mathbb{R} .
$$

В дискретном случае как теорема 1.1, так и теорема 1.2 известны. Дискретный вариант теоремы 1.1 принадлежит Гохбергу и Лереру [15], а теоремы $1.2-$ Ацмону [1]. В работе [15] теорема 1.2 фигурирует в несколько иной форме, использующей для матричных многочленов уравнения другого типа, чем уравнение (1.8).

Теорема 1.2 будет доказана в следующем параграфе, а теорема 1.1 - в последнем параграфе с использованием теоремы 1.2 и основного результата работы [12].

Дополнительные ссылки и дальнейшие комментарии. Ортогональные функции Крейна участвуют и в другой замечательной теореме, которая 
связывает число нулей ортогональной функции с числом отрицательных собственных значений интегрального оператора, заданного левой частью уравнения (1.2). Точнее, для матричных функций справедливо следующее утверждение. Если функция $\Phi$, заданная формулой (1.5), есть ортогональная матричная функция Крейна, порожденная некоторой эрмитовой матричной функцией $k$ из $L_{1}^{n \times n}[-\omega, \omega]$, то соответствующий интегральный оператор $T$ в пространстве $L_{1}^{n}[0, \omega]$, заданный формулой

$$
(T f)(t)=f(t)-\int_{0}^{\omega} k(t-s) f(s) d s, \quad 0 \leqslant t \leqslant \omega,
$$

обратим, и число нулей определителя $\operatorname{det} \Phi(\lambda)$ в верхней полуплоскости равно числу отрицательных собственных значений этого оператора. В скалярном случае этот результат восходит к работе Крейна и Лангера [22], а в матричном случае он содержится в [7] (см. также [6, гл. 8]) и, с другим доказательством, в [5].

Указанные выше результаты имеют и дискретные аналоги. Для скалярного случая они содержатся в [20], а для матричного - в [2] и [15].

Отметим также, что необходимое и достаточное условие для того, чтобы $\Phi$ была ортогональной матричной функцией Крейна, можно сформулировать в терминах симметрических разложений функции $\Phi(\lambda) \Phi(\bar{\lambda})^{*}$ на взаимно простые множители. В этом случае производящую функцию можно получить, используя операторный аналог результантной матрицы; см. по этому поводу теорему 5.8 из [11] (и теорему 5.1 из [15] для дискретного случая).

В заключение скажем несколько слов о приложениях. Ортогональные функции Крейна, как скалярные, так и матричные, играют важную роль в различных разделах анализа и математической теории систем и в разнообразных инженерных задачах. Начиная с последних, отметим, что в работах [16] и [17] (см. также [24]) дана любопытная интерпретация ортогональных функций Крейна как преобразований Фурье процесса обновления для стационарного процесса с ковариацией $\delta(t-s)+k(t-s)$. В статье Крейна и Лангера [22], опубликованной в 1985 г., ортогональные функции играют ключевую роль в решении различных задач расширения и продолжения; см. по этому поводу также статью [23], где фигурируют матричные функции. У решений этих задач продолжения имеются важные приложения в теории оценивания и предсказания для случайных процессов (см., например, [4] и имеющиеся там ссылки). Кроме того, как показано в [22, разд. 12], функции Крейна играют существенную роль в решении обратной спектральной задачи для уравнений Штурма-Лиувилля. Говоря более общо, ортогональные матричные функции Крейна используются при изучении канонических дифференциальных уравнений, в том числе так называемых дифференциальных систем Крейна (см., например, [19], [3], [25] и библиографию в последней из этих работ). Связь с каноническими системами возникает, если длину $\omega$ отрезка в формуле (1.1) рассматривать как вторую независимую переменную. Ортогональные матричные функции Крейна существенным образом возникают и при анализе современных задач о пополнении (см. [5]).

Некоторые обозначения и терминология. Пусть $X$ - матрица размера $k \times \ell$. Тогда через $X^{\top}$ обозначается транспонированная матрица, через $X^{*}-$ эрмитово-сопряженная матрица, а через $\bar{X}$ - матрица размера $k \times \ell$, которая получается, если каждый элемент матрицы $X$ заменить его комплексносопряженным. Таким образом, $\bar{X}=\left(X^{\top}\right)^{*}=\left(X^{*}\right)^{\top}$. Мы отождествляем вектор 
$x \in \mathbb{C}^{n}$ с матрицей размера $n \times 1, j$-й элемент которой равен $j$-й координате вектора $x$ в стандартном базисе пространства $\mathbb{C}^{n}$.

Преобразование Фурье матричной функции $h$ из пространства $L_{1}^{n \times n}(\mathbb{R})$ будет обозначаться через $\hat{h}$, т. е.

$$
\hat{h}(\lambda)=\int_{-\infty}^{\infty} e^{i \lambda s} h(s) d s, \quad \lambda \in \mathbb{R} .
$$

Всюду в работе используется соглашение, в соответствии с которым функция на конечном отрезке рассматривается как функция на всей прямой, доопределенная вне этого отрезка нулем.

\section{§2. Доказательство теоремы 1.2}

В этом параграфе мы докажем теорему 1.2. Разобьем доказательство на две части.

Часть 1. Пусть $\Phi$ - ортогональная функция Крейна вида $(1.5)$, порожденная эрмитовой функцией $k \in L_{1}^{n \times n}[-\omega, \omega]$. Мы должны показать, что у уравнения (1.8) есть регулярная пара решений $U, V$, т. е. пара решений вида (1.9).

Пусть $r$ есть $n \times n$-матричная функция, задаваемая формулой

$$
\varphi(t)-\int_{-\infty}^{\infty} k(t-s) \varphi(s) d s=r(t), \quad t \in \mathbb{R} .
$$

Здесь мы воспользовались упомянутым выше соглашением, в соответствии с которым функция на конечном отрезке рассматривается как функция на всей прямой, доопределенная вне этого отрезка нулем. Поскольку $\Phi$ - ортогональная функция Крейна, порожденная функцией $k$, то функция $\varphi$ удовлетворяет уравнению (1.6) и потому $r(t)=k(t)$ при $0 \leqslant t \leqslant \omega$. Так как функция $\varphi$ сосредоточена на отрезке $[0, \omega]$, а $k$ - на отрезке $[-\omega, \omega]$, то из формулы $(2.1)$ следует, что функция $r$ сосредоточена на отрезке $[-\omega, 2 \omega]$. Зададим функции $u \in L_{1}^{n \times n}[0, \omega)$ и $v \in L_{1}^{n \times n}[\omega, 2 \omega]$ формулами

$$
u(t)=r(-t)^{*}-k(-t)^{*} \quad(0 \leqslant t \leqslant \omega), \quad v(t)=-r(t) \quad(\omega \leqslant t \leqslant 2 \omega) .
$$

Тогда (опять рассматривая функцию на конечном отрезке как функцию на всей прямой, доопределенную вне этого отрезка нулем) мы имеем

$$
r(t)=u(-t)^{*}+k(t)-v(t), \quad \infty<t<\infty .
$$

Используя формулу (2.1) и переходя к преобразованиям Фурье в (2.2), мы получаем, что

$$
\hat{\varphi}(\lambda)-\hat{k}(\lambda) \hat{\varphi}(\lambda)=\hat{k}(\lambda)+\hat{u}(\bar{\lambda})^{*}-\hat{v}(\lambda), \quad \lambda \in \mathbb{R} .
$$

Определим теперь функцию $F$ формулой

$$
F(\lambda)=I_{n}-\int_{-\omega}^{\omega} e^{i \lambda t} k(t) d t, \quad \lambda \in \mathbb{C} .
$$

Поскольку функция $k$ сосредоточена на отрезке $[-\omega, \omega]$, то $F(\lambda)=I_{n}-\hat{k}(\lambda)$, и мы видим, что соотношение (2.3) можно переписать в виде

$$
F(\lambda) \Phi(\lambda)=U(\bar{\lambda})^{*}-V(\lambda), \quad \lambda \in \mathbb{C},
$$


где

$$
U(\lambda)=I_{n}+\int_{0}^{\omega} e^{i \lambda t} u(t) d t, \quad V(\lambda)=\int_{\omega}^{2 \omega} e^{i \lambda t} v(t) d t .
$$

Нужно показать, что эти $U$ и $V$ удовлетворяют уравнению (1.8).

Нам будет удобно воспользоваться алгеброй Винера $\mathscr{W} n \times n$-матричных функций на вещественной прямой. Напомним, что

$$
\mathscr{W}=\left\{H \mid H(\lambda)=M+\int_{-\infty}^{\infty} e^{i \lambda t} h(t) d t, M \in \mathbb{C}^{n \times n}, h \in L_{1}^{n \times n}(\mathbb{R})\right\} .
$$

Для любого элемента $H \in \mathscr{W}$ определим функцию $H^{*}$ формулой $H^{*}(\lambda)=$ $H(\lambda)^{*}, \lambda \in \mathbb{R}$. Тогда $H^{*} \in \mathscr{W}$, причем отображение ${ }^{*}$ - инволюция в алгебре $\mathscr{W}$. Элемент $H \in \mathscr{W}$ называется самосопряжсенным, если $H^{*}=H$. Если $\mathscr{V} \subset \mathscr{W}$ - произвольное подмножество, то через $\mathscr{V}^{*}$ обозначим множество всех элементов $H^{*}$, где $H \in \mathscr{V}$. Нам потребуются также следующие подпространства B $\mathscr{W}:$

$$
\begin{aligned}
& \mathscr{W}_{1}=\left\{H \mid H(\lambda)=\int_{\omega}^{\infty} e^{i \lambda t} h(t) d t, h \in L_{1}^{n \times n}[\omega, \infty)\right\}, \\
& \mathscr{W}_{2}=\left\{H \mid H(\lambda)=M+\int_{0}^{\omega} e^{i \lambda t} h(t) d t, M \in \mathbb{C}^{n \times n}, h \in L_{1}^{n \times n}[0, \omega]\right\}, \\
& \mathscr{W}_{3}^{0}=\left\{H \mid H(\lambda)=\int_{-\omega}^{0} e^{i \lambda t} h(t) d t, h \in L_{1}^{n \times n}[-\omega, 0]\right\}, \\
& \mathscr{W}_{4}=\left\{H \mid H(\lambda)=\int_{-\infty}^{-\omega} e^{i \lambda t} h(t) d t, h \in L_{1}^{n \times n}(-\infty,-\omega]\right\} .
\end{aligned}
$$

Они задают разложение

$$
\mathscr{W}=\mathscr{W}_{1} \oplus \mathscr{W}_{2} \oplus \mathscr{W}_{3}^{0} \oplus \mathscr{W}_{4}
$$

пространства $\mathscr{W}$. Заметим, что $\mathscr{W}_{1}^{*}=\mathscr{W}_{4}$ и $\mathscr{W}_{2}^{*}=\mathscr{W}_{3}$, где

$$
\mathscr{W}_{3}=\left\{H \mid H(\lambda)=M+\int_{-\omega}^{0} e^{i \lambda t} h(t) d t, M \in \mathbb{C}^{n \times n}, h \in L_{1}^{n \times n}[-\omega, 0]\right\} .
$$

Для этих подпространств выполнен ряд правил умножения. Например,

$$
\mathscr{W}_{2} \mathscr{W}_{1} \subset \mathscr{W}_{1}, \quad \mathscr{W}_{2} \mathscr{W}_{2} \subset \mathscr{W}_{1} \oplus \mathscr{W}_{2}, \quad \mathscr{W}_{3} \mathscr{W}_{1} \subset \mathscr{W}_{1} \oplus \mathscr{W}_{2} .
$$

Напомним, что функция $k$ эрмитова. Поэтому из определения (2.4) вытекает, что $F(\bar{\lambda})^{*}=F(\lambda)$ для всех $\lambda \in \mathbb{C}$. В частности, $F(\lambda)^{*}=F(\lambda)$ при $\lambda \in \mathbb{R}$. Следовательно, $F^{*}=F$, т. е. $F-$ самосопряженный элемент алгебры $\mathscr{W}$. Опуская переменную $\lambda$, можно переписать соотношение $(2.5)$ в виде $F \Phi=U^{*}-V$, где все функции рассматриваются только на $\mathbb{R}$. Используя тот факт, что $F^{*}=F$, получаем $\Phi^{*} F=U-V^{*}$. Отсюда следует, что

$$
\begin{aligned}
U \Phi+\Phi^{*} V & =U \Phi+\Phi^{*} V+V^{*} \Phi-V^{*} \Phi \\
& =\Phi^{*} V+V^{*} \Phi+\left(U-V^{*}\right) \Phi=\Phi^{*} V+V^{*} \Phi+\Phi^{*} F \Phi .
\end{aligned}
$$


Функции $\Phi, U$ и $V$ лежат в алгебре $\mathscr{W}$. Точнее, $\Phi, U \in \mathscr{W}_{2}$ и $V \in \mathscr{W}_{1}$. Поскольку элементы $\Phi^{*} V+V^{*} \Phi$ и $\Phi^{*} F \Phi$ являются самосопряженными в $\mathscr{W}$, то самосопряжен и элемент $U \Phi+\Phi^{*} V$. Но

$$
U \Phi+\Phi^{*} V \in \mathscr{W}_{2} \mathscr{W}_{2}+\mathscr{W}_{3} \mathscr{W}_{1} \subset \mathscr{W}_{+}+\mathscr{W}_{+} \subset \mathscr{W}_{+}
$$

где $\mathscr{W}_{+}=\mathscr{W}_{1} \oplus \mathscr{W}_{2}$, т. е.

$$
\mathscr{W}_{+}=\left\{H \mid H(\lambda)=M+\int_{0}^{\infty} e^{i \lambda t} h(t) d t, M \in \mathbb{C}^{n \times n}, h \in L_{1}^{n \times n}[0, \infty)\right\} .
$$

Таким образом, из самосопряженности элемента $U \Phi+\Phi^{*} V$ вытекает, что $U \Phi+$ $\Phi^{*} V \in \mathscr{W}_{+} \cap\left(\mathscr{W}_{+}\right)^{*}$. Мы заключаем, что $U \Phi+\Phi^{*} V-$ постоянная функция, так что

$$
U(\lambda) \Phi(\lambda)+\Phi(\bar{\lambda})^{*} V(\lambda)=\lim _{\lambda \rightarrow \infty}(U(\lambda) \Phi(\lambda)+\Phi(\bar{\lambda}) V(\lambda))=I_{n} .
$$

Поэтому $U, V$ - регулярная пара решений уравнения (1.8).

Часть 2. В этой части предполагается, что $U, V$ - регулярная пара решений уравнения (1.8), т. е. $U$ и $V$ удовлетворяют этому уравнению и имеют вид (1.9). Нужно доказать, что $\Phi$ - ортогональная функция Крейна. Заметим, что $U$ и $V$ принадлежат алгебре Винера $\mathscr{W}$.

Прежде всего покажем, что $\operatorname{det} \Phi(\lambda) \neq 0$ для всех $\lambda \in \mathbb{R}$. Зафиксируем число $\lambda \in \mathbb{R}$ и предположим, что $\operatorname{det} \Phi(\lambda)=0$. Тогда существует такой ненулевой вектор $x \in \mathbb{C}^{n}$, что $\Phi(\lambda) x=0$. Так как $\lambda$ вещественно $(\lambda=\bar{\lambda})$, то $x^{*} \Phi(\bar{\lambda})^{*}=0$. Таким образом, из уравнения (1.8) вытекает, что $x^{*} x=0$, чего не может быть, поскольку $x \neq 0$. Итак, $\operatorname{det} \Phi(\lambda) \neq 0$, что и требовалось.

Поскольку $\Phi$ принадлежит алгебре Винера, то из результата, сформулированного в предыдущем абзаце, вытекает (в силу теоремы Винера), что элемент $\Phi$ обратим в $\mathscr{W}$. Определим функцию $F$ формулой

$$
F(\lambda)=\left(U(\bar{\lambda})^{*}-V(\lambda)\right) \Phi(\lambda)^{-1}, \quad \lambda \in \mathbb{C} .
$$

Тогда она лежит в алгебре Винера. Докажем, что элемент $F$ самосопряжен в $\mathscr{W}$, т. е. $F=F^{*}$.

Для этого прежде всего заметим, что уравнение (1.8) можно переписать в виде $U \Phi+\Phi^{*} V=E$, где элемент $E \in \mathscr{W}$ - функция, тождественно равная $I_{n}$. Переходя к сопряженным элементам, получаем $\Phi^{*} U^{*}+V^{*} \Phi=E$, поскольку $E^{*}=E$. Так как правая часть обоих тождеств равна $E$, то $U \Phi+\Phi^{*} V=\Phi^{*} U^{*}+$ $V^{*} \Phi$, так что

$$
\left(U-V^{*}\right) \Phi=\Phi^{*}\left(U^{*}-V\right) .
$$

Умножив это тождество справа на $\Phi^{-1}$ и слева на $\left(\Phi^{*}\right)^{-1}$, мы получим $\left(U^{*}-\right.$ $V) \Phi^{-1}=\left(\Phi^{*}\right)^{-1}\left(U-V^{*}\right)$. Но $\left(\Phi^{*}\right)^{-1}=\left(\Phi^{-1}\right)^{*}$, так что

$$
F=\left(U^{*}-V\right) \Phi^{-1}=\left(\Phi^{-1}\right)^{*}\left(U-V^{*}\right)=F^{*} .
$$

Таким образом, $F$ - самосопряженный элемент алгебры $\mathscr{W}$.

Из определения элемента $F$ видно, что

$$
\lim _{\lambda \in \mathbb{R}, \lambda \rightarrow \infty} F(\lambda)=\lim _{\lambda \in \mathbb{R}, \lambda \rightarrow \infty}\left(U(\bar{\lambda})^{*}-V(\lambda)\right) \Phi(\lambda)^{-1}=I_{n} .
$$

Отсюда следует, что

$$
F(\lambda)=I_{n}-\int_{-\infty}^{\infty} e^{i \lambda t} \ell(t) d t, \quad \lambda \in \mathbb{R}, \text { для некоторого } \ell \in L_{1}^{n \times n}(\mathbb{R}) .
$$


Так как $F$ самосопряжен в $\mathscr{W}$, то $\ell$ эрмитов, т. е. удовлетворяет соотношению $\ell(t)^{*}=\ell(-t)$ для всех $t \in \mathbb{R}$. Пусть $k-$ сужение функции $\ell$ на отрезок $[-\omega, \omega]$. Тогда $k \in L_{1}^{n \times n}[-\omega, \omega]$ и элемент $k$ эрмитов. Мы покажем, что $\Phi$ - ортогональная функция Крейна, порожденная функцией $k$.

Заметим, что

$$
F(\lambda)=-L_{1}(\lambda)+F_{0}(\lambda)-L_{4}(\lambda), \quad \lambda \in \mathbb{R},
$$

где

$$
\begin{gathered}
F_{0}(\lambda)=I-\int_{-\omega}^{\omega} e^{i \lambda t} k(t) d t, \quad \lambda \in \mathbb{C}, \\
L_{1}(\lambda)=\int_{\omega}^{\infty} e^{i \lambda t} \ell(t) d t, \quad L_{4}(\lambda)=\int_{-\infty}^{-\omega} e^{i \lambda t} \ell(t) d t \quad \lambda \in \mathbb{R} .
\end{gathered}
$$

Отсюда следует, что

$$
\begin{aligned}
F_{0}(\lambda) \Phi(\lambda) & =\left(F(\lambda)+L_{1}(\lambda)+L_{4}(\lambda)\right) \Phi(\lambda) \\
& =U(\lambda)^{*}-V(\lambda)+L_{1}(\lambda) \Phi(\lambda)+L_{4}(\lambda) \Phi(\lambda), \quad \lambda \in \mathbb{R} .
\end{aligned}
$$

Заметим, что $L_{1} \in \mathscr{W}_{1}$. Поэтому $L_{1} \Phi \in \mathscr{W}_{1} \mathscr{W}_{2} \subset \mathscr{W}_{1}$, т. е. для некоторой функции $h_{1} \in L_{1}^{n \times n}[\omega, \infty)$ выполнено соотношение

$$
L_{1}(\lambda) \Phi(\lambda)=\int_{\omega}^{\infty} e^{i \lambda t} h_{1}(t) d t, \quad \lambda \in \mathbb{R} .
$$

Так как $L_{4} \in \mathscr{W}_{4}$, то $L_{4} \Phi \in \mathscr{W}_{4} \mathscr{W}_{2} \subset \mathscr{W}_{-}^{0}$, где $\mathscr{W}_{-}^{0}=\mathscr{W}_{3}^{0} \oplus \mathscr{W}_{4}$. Таким образом,

$$
L_{4}(\lambda) \Phi(\lambda)=\int_{-\infty}^{0} e^{i \lambda t} h_{-}(t) d t, \quad \lambda \in \mathbb{R},
$$

для некоторого $h_{-} \in L_{1}^{n \times n}(-\infty, 0]$. Теперь положим

$$
r_{-}(t)=u(-t)^{*}+h_{-}(t) \quad(t \leqslant 0), \quad r_{1}=-v(t)+h_{1}(t) \quad(t \geqslant \omega) .
$$

Используя предыдущие формулы, видим, что

$$
\begin{aligned}
\hat{\varphi}(\lambda)-\hat{k}(\lambda) \hat{\varphi}(\lambda) & =-F_{0}(\lambda)+F_{0}(\lambda) \Phi(\lambda) \\
& =-F_{0}(\lambda)+U(\lambda)^{*}-V(\lambda)+L_{1}(\lambda) \Phi(\lambda)+L_{4}(\lambda) \Phi(\lambda) \\
& =\hat{k}(\lambda)+\hat{r_{-}}(\lambda)+\hat{r_{1}}(\lambda), \quad \lambda \in \mathbb{R} .
\end{aligned}
$$

Переходя к обратному преобразованию Фурье, получаем

$$
\varphi(t)-\int_{-\infty}^{\infty} k(t-s) \varphi(s)=k(t)+r_{-}(t)+r_{1}(t), \quad t \in \mathbb{R} .
$$

Напомним, что носители функций $\varphi, r_{-}$и $r_{1}$ содержатся в интервалах $[0, \omega)$, $(-\infty, 0]$ и $[\omega, \infty)$ соответственно. Поэтому сужение на интервал $0 \leqslant t \leqslant \omega$ дает

$$
\varphi(t)-\int_{0}^{\omega} k(t-s) \varphi(s)=k(t), \quad 0 \leqslant t \leqslant \omega .
$$

Итак, $\Phi$ - ортогональная функция Крейна, порожденная функцией $k$. 
Как мы показали выше, функция $k$ есть сужение функции $\ell$ на отрезок $[-\omega, \omega]$. Другими словами, $k-$ сужение на отрезок $[-\omega, \omega]$ обратного преобразования Фурье матричной функции (1.10). Итак, мы одновременно доказали последнее утверждение теоремы 1.2.

Замечание. Судя по приведенному выше доказательству, можно ожидать, что теорема 1.2 имеет абстрактный вариант, использующий лишь структуру, возникающую из ленточного метода (cp. [8, гл. XXXIV]) для соответствующей алгебры функций. Мы вернемся к этой теме в одной из будущих публикаций.

\section{§3. Доказательство теоремы 1.1}

В этом параграфе мы докажем теорему 1.1. С учетом теоремы 1.2 достаточно показать, что следующие два утверждения эквивалентны:

(a) у уравнения (1.8) есть регулярная пара решений $U, V$;

(b) у определителя $\operatorname{det} \Phi(\lambda)$ нет вещественных нулей, а для любой симметричной пары $\lambda_{0}, \bar{\lambda}_{0}$ его нулей выполнено соотношение

$$
\sum_{j=0}^{k}\left\langle\varphi_{k-j}, \psi_{j}\right\rangle_{\mathbb{C}^{n}}=0, \quad k=0,1, \ldots, \min \{p, q\}-1,
$$

где $\varphi_{0}, \varphi_{1}, \ldots, \varphi_{p-1}$ и $\psi_{0}, \psi_{1}, \ldots, \psi_{q-1}$ - произвольные жордановы цепочки для $\Phi$ в точках $\lambda_{0}$ и $\bar{\lambda}_{0}$ соответственно.

Доказательство этой эквивалентности разобьем на две части. Во второй части мы воспользуемся теоремой 1.1 из [12]. В этой теореме рассматривается матричное функциональное уравнение вида

$$
X(\lambda) \mathscr{B}(\lambda)+\mathscr{D}(\lambda) Y(\lambda)=G(\lambda), \quad \lambda \in \mathbb{C},
$$

где коэффициенты $\mathscr{B}$ и $\mathscr{D}$ суть $n \times n$-матричные целые функции, задаваемые формулами

$$
\mathscr{B}(\lambda)=I_{n}+\int_{-\omega}^{0} e^{i \lambda t} b(t) d t, \quad \mathscr{D}(\lambda)=I_{n}+\int_{0}^{\omega} e^{i \lambda t} d(t) d t .
$$

Здесь $b \in L_{1}^{n \times n}[-\omega, 0]$ и $d \in L_{1}^{n \times n}[0, \omega]$. Предполагается, что правая часть известна и имеет вид

$$
G(\lambda)=\int_{-\omega}^{\omega} e^{i \lambda t} g(t) d t, \quad \text { где } g \in L_{1}^{n \times n}[-\omega, \omega] .
$$

Задача состоит в том, чтобы найти $n \times n$-матричные целые функции $X$ и $Y$ вида

$$
X(\lambda)=\int_{0}^{\omega} e^{i \lambda t} x(t) d t, \quad Y(\lambda)=\int_{-\omega}^{0} e^{i \lambda t} y(t) d t,
$$

где $x \in L_{1}^{n \times n}[0, \omega]$ и $y \in L_{1}^{n \times n}[-\omega, 0]$, удовлетворяющие уравнению (3.1). Теорема 1.1 из [12] гласит:

Теорема 3.1. Для существования функиий $X, Y$ вида (3.4), удовлетворяющих уравнению (3.1), необходимо и достаточно, чтобы для каждого общего нуля $\lambda_{0}$ определителей $\operatorname{det} \mathscr{B}(\lambda) u \operatorname{det} \mathscr{D}(\lambda)$ было выполнено следующее условие: 
если $\varphi$ - корневая функиия порядка р для $\mathscr{B}(\lambda)$ в точке $\lambda_{0}$, а $\psi$ - корневая функиия порядка q для $\mathscr{D}(\lambda)^{\top}$ в точке $\lambda_{0}$, то функиия

$$
\psi(\lambda)^{\top} G(\lambda) \varphi(\lambda)
$$

имеет в точке $\lambda_{0}$ нуль порядка не ниже $\min \{q, p\}$.

Мы вернемся к этой теореме во второй части доказательства.

Часть 1. Пусть выполнено условие (а). Итак, существуют функции $U$ и $V$ вида (1.9), удовлетворяющие уравнению (1.8). Как мы видели во втором абзаце части 2 доказательства, приведенного в предыдущем параграфе, отсюда следует, что определитель $\operatorname{det} \Phi(\lambda)$ не имеет нулей на вещественной оси.

Пусть $\varphi_{0}, \varphi_{1}, \ldots, \varphi_{p-1}$ - жорданова цепочка для $\Phi$ в точке $\lambda_{0}$, а $\psi_{0}, \psi_{1}, \ldots$, $\psi_{q-1}$ - жорданова цепочка для $\Phi$ в точке $\bar{\lambda}_{0}$. Рассмотрим $\mathbb{C}^{n}$-значные функции

$$
\varphi(\lambda)=\sum_{i=0}^{p-1}\left(\lambda-\lambda_{0}\right)^{i} \varphi_{i}, \quad \psi(\lambda)=\sum_{j=0}^{q-1}\left(\lambda-\bar{\lambda}_{0}\right)^{j} \psi_{j} .
$$

Так как $\varphi_{0}, \varphi_{1}, \ldots, \varphi_{p-1}$ - жорданова цепочка для $\Phi$ в точке $\lambda_{0}$, то $\Phi(\lambda) \varphi(\lambda)$ имеет в точке $\lambda_{0}$ нуль порядка не ниже $p$. Аналогично, $\Phi(\lambda) \psi(\lambda)$ имеет в точке $\bar{\lambda}_{0}$ нуль порядка не ниже $q$. Из последнего утверждения следует, что $\psi(\bar{\lambda})^{*} \Phi(\bar{\lambda})^{*}$ имеет в точке $\lambda_{0}$ нуль порядка не ниже $q$. Воспользуемся теперь тем, что $U$ и $V$ удовлетворяют уравнению (1.8). Умножая его слева на $\psi(\bar{\lambda})^{*}$ и справа на $\varphi(\lambda)$, преобразуем правую часть к виду $\psi(\bar{\lambda})^{*} \varphi(\lambda)$. Но после этих умножений левая часть имеет в точке $\lambda_{0}$ нуль порядка не ниже $\min \{q, p\}$. Поэтому в точке $\lambda_{0}$ функция $\psi(\bar{\lambda})^{*} \varphi(\lambda)$ обязана иметь нуль порядка не ниже $\min \{q, p\}$. Последнее утверждение эквивалентно соотношениям (1.7). Таким образом, условие (b) выполнено.

Часть 2. Здесь мы докажем обратную импликацию. Итак, предположим, что выполнено условие (b).

Необходимо доказать существование функций $U$ и $V$ вида (1.9), удовлетворяющих уравнению (1.8). Для этого воспользуемся теоремой 3.1. В качестве первого шага перепишем уравнение (1.8) в таком виде, чтобы эту теорему можно было применить. Положим

$$
x(t)=u(\omega-t), \quad y(t)=v(\omega-t), \quad \beta(t)=\varphi(-t), \quad \delta(t)=\varphi(t)^{*} .
$$

Здесь $t \in \mathbb{R}$ и, как и в предыдущем параграфе, мы используем соглашение, в соответствии с которым функция на конечном отрезке рассматривается как функция на всей прямой, доопределенная вне этого отрезка нулем. Заметим, что носители функций $x$ и $\delta$ содержатся в отрезке $[0, \omega]$, а носители функций $y$ и $\beta-$ в отрезке $[-\omega, 0]$. Теперь определим функции

$$
\begin{array}{ll}
X(\lambda)=\int_{0}^{\omega} e^{i \lambda t} x(t) d t, & Y(\lambda)=\int_{-\omega}^{0} e^{i \lambda t} y(t) d t \\
\mathscr{D}(\lambda)=I_{n}+\int_{0}^{\omega} e^{i \lambda t} \delta(t) d t, & \mathscr{B}(\lambda)=I_{n}+\int_{-\omega}^{0} e^{i \lambda t} \beta(t) d t .
\end{array}
$$


Непосредственное вычисление показывает, что

$$
\begin{gathered}
U(\lambda)=I_{n}+e^{i \lambda \omega} X(-\lambda), \quad V(\lambda)=e^{i \lambda \omega} Y(-\lambda), \\
\Phi(\lambda)=\mathscr{B}(-\lambda), \quad \Phi(\bar{\lambda})^{*}=\mathscr{D}(-\lambda), \quad \lambda \in \mathbb{C} .
\end{gathered}
$$

Используя эти тождества и заменяя $\lambda$ на $-\lambda$, можно переписать уравнение $(1.8)$ в следующем эквивалентном виде:

$$
X(\lambda) \mathscr{B}(\lambda)+\mathscr{D}(\lambda) Y(\lambda)=e^{i \lambda \omega} I_{n}-e^{i \lambda \omega} \mathscr{B}(\lambda), \quad \lambda \in \mathbb{C} .
$$

Таким образом, достаточно показать, что у уравнения (3.8) есть пара решений $X, Y$ вида (3.6). Для этого воспользуемся теоремой 3.1. Нам нужно лишь показать, что для этого уравнения выполнено достаточное условие теоремы 3.1.

Итак, пусть $\lambda_{0}-$ общий нуль определителей $\operatorname{det} \mathscr{B}(\lambda)$ и $\operatorname{det} \mathscr{D}(\lambda), \varphi-$ корневая функция порядка $p$ для $\mathscr{B}(\lambda)$ в точке $\lambda_{0}$, а $\psi-$ корневая функция порядка $q$ для $\mathscr{D}(\lambda)^{\top}$ в той же точке. Положим

$$
G(\lambda)=e^{i \lambda \omega} I_{n}-e^{i \lambda \omega} \mathscr{B}(\lambda), \quad \lambda \in \mathbb{C} .
$$

Для завершения доказательства остается показать, что если выполнено условие $(\mathrm{b})$, то функция $\psi(\lambda)^{\top} G(\lambda) \varphi(\lambda)$ имеет в точке $\lambda_{0}$ нуль порядка не ниже $\min \{q, p\}$. Поскольку у $\mathscr{B}(\lambda) \varphi(\lambda)$ в точке $\lambda_{0}$ имеется нуль порядка $p$, то же самое верно и для $\psi(\lambda)^{\top} e^{i \lambda \omega} \mathscr{B}(\lambda) \varphi(\lambda)$. Итак, нужно проверить, что $\psi(\lambda)^{\top} e^{i \lambda \omega} \varphi(\lambda)$ имеет нуль порядка не ниже $\min \{q, p\}$ в точке $\lambda_{0}$. Множитель $e^{i \lambda \omega}$ не играет роли, так что мы должны показать, что

$$
\psi(\lambda)^{\top} \varphi(\lambda) \text { имеет нуль порядка не ниже } \min \{q, p\} \text { в точке } \lambda_{0} \text {. }
$$

Условие (3.9) можно переписать в терминах тейлоровских разложений

$$
\varphi(\lambda)=\sum_{j=0}^{\infty} \varphi_{j}\left(\lambda-\lambda_{0}\right)^{j}, \quad \psi(\lambda)=\sum_{j=0}^{\infty} \psi_{j}\left(\lambda-\lambda_{0}\right)^{j}
$$

функций $\varphi$ и $\psi$ в окрестности точки $\lambda_{0}$ в виде

$$
\sum_{j=0}^{k} \psi_{j}^{\top} \varphi_{k-j}=0, \quad k=0, \ldots, \min \{q, p\}-1 .
$$

Теперь напомним, что $\mathscr{B}(\lambda)=\Phi(-\lambda)$ и $\mathscr{D}(\lambda)=\Phi(-\bar{\lambda})^{*}$ для всех $\lambda \in \mathbb{C}$. Поскольку $\varphi-$ корневая функция порядка $p$ для $\mathscr{B}(\lambda)$ в точке $\lambda_{0}$, то векторы $\varphi_{0},(-1) \varphi_{1}, \ldots,(-1)^{p-1} \varphi_{p-1}$ образуют жорданову цепочку для $\Phi$ в точке $-\lambda_{0}$. Аналогично, поскольку $\psi$ - корневая функция порядка $q$ для $\mathscr{D}(\lambda)^{\top}$ в точке $\lambda_{0}$, мы делаем вывод, что векторы $\bar{\psi}_{0},(-1) \bar{\psi}_{1}, \ldots,(-1)^{q-1} \bar{\psi}_{q-1}$ образуют жорданову цепочку для $\Phi$ в точке $-\bar{\lambda}_{0}$. В соответствии с условием (b) отсюда следует, что

$$
\sum_{j=0}^{k}\left\langle(-1)^{k-j} \varphi_{k-j},(-1)^{j} \bar{\psi}_{j}\right\rangle=0, \quad k=0, \ldots, \min \{q, p\}-1 .
$$

Теперь заметим, что

$$
\psi_{j}^{\top} \varphi_{k-j}=(-1)^{k}(-1)^{j}\left(\bar{\psi}_{j}\right)^{*}(-1)^{k-j} \varphi_{k-j}=(-1)^{k}\left\langle(-1)^{k-j} \varphi_{k-j},(-1)^{j} \bar{\psi}_{j}\right\rangle_{\mathbb{C}^{n}}
$$


при $j=0, \ldots, k \leqslant \min \{q, p\}-1$. Значит, при $k=0, \ldots, \min \{q, p\}-1$ мы получаем

$$
\sum_{j=0}^{k} \psi_{j}^{\top} \varphi_{k-j}=(-1)^{k} \sum_{j=0}^{k}\left\langle(-1)^{k-j} \varphi_{k-j},(-1)^{j} \bar{\psi}_{j}\right\rangle=0 .
$$

Итак, условие (3.10) установлено, и для завершения доказательства условия (а) остается применить теорему 3.1 .

\section{ЛитеРАТУРА}

[1] A. Atzmon, n-orthogonal operator polynomials, in: Orthogonal Matrix-Valued Polynomials and Applications (Tel Aviv, 1987-88), vol. 34 (ed. I. Gohberg), Birkhäuser, Basel, 1988, 47-63.

[2] D. Alpay, I. Gohberg, On orthogonal matrix polynomials, in: Orthogonal MatrixValued Polynomials and Applications (Tel Aviv, 1987-88), vol. 34 (ed. I. Gohberg), Birkhäuser, Basel, 1988, 25-46.

[3] S. A. Denisov, To the spectral theory of Krein systems, Integral Equations Operator Theory, 42:2 (2002), 166-173.

[4] H. Dym, On reproducing kernels and the continuous covariance extension problem, in: Analysis and Partial Differential Equations, Lecture Notes in Pure and Appl. Math., vol. 122 (ed. C. Sadosky), Marcel Dekker, New York, 1990, 427-482.

[5] H. Dym, On the zeros of some continuous analogues of matrix polynomials and a related extension problem with negative squares, Comm. Pure Appl. Math., 47:2 (1994), $207-256$.

[6] R. L. Ellis, I. Gohberg, Orthogonal Systems and Convolution Operators, Oper. Theory Adv. Appl., vol. 140, Birkhäuser, Basel, 2003.

[7] R. L. Ellis, I. Gohberg, D. C. Lay, Distribution of zeros of matrix-valued continuous analogues of orthogonal polynomials, in: Continuous and Discrete Fourier Transforms, Extension Problems and Wiener-Hopf Equations, Oper. Theory Adv. Appl., vol. 58 (ed. I. Gohberg), Birkhäuser, Basel, 1992, 26-70.

[8] I. Gohberg, S. Goldberg, M. A. Kaashoek, Classes of Linear Operators. Vol. II, Oper. Theory Adv. Appl., vol. 63, Birkhäuser, Basel, 1993.

[9] I. Gohberg, I. Haimovici, M. A. Kaashoek, L. Lerer, The Bezout integral operator: main property and underlying abstract scheme, in: The State Space Method. Generalizations and Applications, Oper. Theory Adv. Appl., vol. 161, Birkhäuser, Basel, 2005, 225270.

[10] И. Ц. Гохберг, Г. Хайниг, О матричных интегралъных операторах на конечном интервале с ядрами, зависящими от разности аргументов, Rev. Roumaine Math. Pures Appl., 20:1 (1975), 55-73.

[11] I. Gohberg, M. A. Kaashoek, L. Lerer, The continuous analogue of the resultant and related convolution operators, in: The Extended Field of Operator Theory, Oper. Theory Adv. Appl., vol. 171 (ed. M. A. Dritschel), Birkhäuser, Basel, 2006, 107-127.

[12] I. Gohberg, M. A. Kaashoek, L. Lerer, On a class of entire matrix function equations, volume dedicated to P. Fuhrmann, Linear Algebra Appl., 2007 (to appear).

[13] I. Gohberg, M. A. Kaashoek, F. van Schagen, Partially Specified Matrices and operators: Classification, Completion, Applications, Oper. Theory Adv. Appl., vol. 79, Birkhäuser, Basel, 1995.

[14] I. Gohberg, M. A. Kaashoek, F. van Schagen, On inversion of convolution integral operators on a finite interval, in: Operator Theoretical Methods and Applications to Mathematical Physics. The Erhard Meister Memorial Volume, Oper. Theory Adv. Appl., vol. 147, Birkhäuser, Basel, 2004, 277-285. 
[15] I. Gohberg, L. Lerer, Matrix generalizations of M. G. Krein theorems on matrix polynomials, in: Orthogonal Matrix-Valued Polynomials and Applications, Oper. Theory Adv. Appl., vol. 34 (ed. I. Gohberg), Birkhäuser, Basel, 1988, 137-202.

[16] by T. Kailath $A$ view of three decades of linear filtering theory, IEEE Trans. Information Theory, IT-20 (1974), 145-181.

[17] T. Kailath, A. Viera, M. Morf, Inverses of Toeplitz operators, innovations and orthogonal polynomials, SIAM Review, 20 (1978), 106-116.

[18] М. Г. Крейн, Континуалъные аналоги предложений о многочленах, ортогональнъх на единичной окружности, Докл. АН СССР, 105:4 (1955), 637-640.

[19] М. Г. Крейн, К теории акселерант и S-матриц, канонических дифференциальнъх систем, Докл. АН СССР, 111 (1956), 1167-1170.

[20] М. Г. Крейн, О расположении корней многочленов, ортогональных на единичной окружности по знакопеременному весу, Теория функций, функц. анализ и приложен., 2 (1966), 131-137.

[21] М. Г. Крейн, Г. Лангер, Континуальные аналоги ортогональных многочленов на единичной окружности по индефинитному весу и связанные с ними проблемы продолжения, Докл. АН СССР, 258 (1981), 537-541.

[22] M. G. Kreǐn, H. Langer, On some continuation problems which are closely related to the theory of operators in spaces $\Pi_{\kappa}$. IV: Continuous analogues of orthogonal polynomials on the unit circle with respect to an indefinite weight and related continuation problems for some classes of functions, J. Operator Theory, 13:2 (1985), 299-417.

[23] М. Г. Крейн, Ф. Э. Мелик-Адамян, Матрично-континуальные аналоги задач Шуpa u Kаратеодори-Тёплица, Изв. АН Арм. ССР, 21:2 (1986), 107-141.

[24] A. Lindquist, On Fredholm integral equations, Toeplitz equations and Kalman-Bucy filtering, Appl. Math. Optim., 1 (1975), 355-373.

[25] L. A. Sakhnovich, On Krein's differential system and its generalization, Integral Equations Operator Theory, 55:4 (2006), 561-572.

School of Mathematical Sciences, Tel-Aviv University

Afdeling Wiskunde, Faculteit der Exacte Wetenschappen,

Поступило в редакцию

Vrije Universiteit, Amsterdam

Department of Mathematics,

Technion - Israel Institute of Technology, Haifa

1 ноября 2006 г. 